https://doi.org/10.15407/ujpe63.11.969

T. ABEBE

Department of Physics, Jimma University

(P. O. Box 378, Jimma, Ethiopia; e-mail: tam1704@gmail.com)

\title{
THE QUANTUM ANALYSIS OF A NONDEGENERATE THREE-LEVEL LASER WITH SPONTANEOUS EMISSION AND NOISELESS VACUUM RESERVOIR
}

\begin{abstract}
The analysis of quantum properties of the cavity light produced by a coherently driven nondegenerate three-level laser possessing an open cavity and coupled to a two-mode vacuum reservoir is presented. The normal ordering of noise operators associated with the vacuum reservoir is considered. Applying the solutions of the equations of evolution for the expectation values of the atomic operators and the quantum Langevin equations for the cavity mode operators, the squeezing properties, entanglement amplification, and the normalized second-order correlation function of the cavity radiation are described. The three-level laser generates squeezed light under certain conditions, with maximum intracavity squeezing being $50 \%$ below the vacuum-state level. Moreover, it is found that the presence of spontaneous emission increases the quadrature squeezing and entanglement and decreses the mean photon number of the two-mode cavity radiation.

Keywords: operator dynamics, quadrature squeezing, second-order correlations, photon entanglement.
\end{abstract}

\section{Introduction}

Entanglement is one of the fundamental tools for the quantum information processing and communication protocols. The generation and manipulation of the entanglement has attracted a great deal of interest with wide applications in quantum teleportation, quantum dense coding, quantum computation, quantum error correction, and quantum cryptography $[1-5]$. Recently, the much attention is given to the generation of a continuous-variable entanglement to manipulate the discrete counterparts and quantum bits and to perform the quantum information processing. In general, the degree of entanglement decreases, when it interacts with the environment. But, the quantum information processing efficiency highly depends on the degree of entanglement. Therefore, it is necessary to generate strongly entangled states which can survive under the external noise.

In general, due to the strong correlation between the cavity modes, a two-mode squeezed state violates certain classical inequalities and then can be used in preparing the Einstein-Podolsky-Rosen (EPR)-type entanglement [6]. The steady state entanglement in a nondegenerate three-level laser has been studied,

(C) T. ABEBE, 2018

ISSN 2071-0194. Ukr. J. Phys. 2018. Vol. 63, No. 11 when the atomic coherence is induced by initially preparing atoms in a coherent superposition of the top an bottom levels [7-13] and when the top and bottom levels of three-level atoms injected into a cavity are coupled by coherent light [14-21]. Moreover, Fesseha studied the quantum properties of the light emitted by three-level atoms available in a closed cavity and pumped to the top level at a constant rate by means of the electron bombardment [22]. Furthermore, he considered the quantum properties of the light generated by a two-level laser in which the twolevel atoms available in a closed cavity are pumped to the upper level by means of the electron bombardment $[23,24]$.

In this paper, we study the squeezing and entanglement properties of the light generated by a coherently driven nondegenerate three-level laser possessing an open cavity and coupled to a two-mode vacuum reservoir via a single-port mirror. In order to carry out our calculation, we put the noise operators associated with the vacuum reservoir in the normal order. First, we will obtain the master equation and the quantum Langevin equations for the cavity mode operators. Then, employing the master equation and the large-time approximation scheme, we obtain the equations of evolution of the expectation 
values of atomic operators. Moreover, we will determine the solutions of the equations of evolution of the expectation values of the atomic operators and the quantum Langevin equations for cavity mode operators. Applying the resulting solutions, we obtain the mean photon number, quadrature squeezing, and entanglement. Furthermore, applying the same solutions, we also obtain the normalized second-order correlation function for the two-mode light.

\section{Master Equation}

Here, $N$ nondegenerate three-level atoms in the cascade configuration are available in an open cavity. The top, intermediate, and bottom levels of the three-level atom are denoted by $|a\rangle_{k},|b\rangle_{k}$, and $|c\rangle_{k}$, respectively. When the atom makes a transition from the level $|a\rangle_{k}$ to $|b\rangle_{k}$ and from the levels $|b\rangle_{k}$ to $|c\rangle_{k}$, two photons with different frequencies are emitted. It is assumed that the cavity mode $a$ is at resonance with the transition $|a\rangle_{k} \rightarrow|b\rangle_{k}$, and the cavity mode $b$ is at resonance with the transition $|b\rangle_{k} \rightarrow|c\rangle_{k}$, with top and bottom levels of the three-level atom coupled by coherent light. The interaction of a nondegenerate three-level atom with the coherent light and with the light modes $a$ and $b$ can be described by the Hamiltonian

$\hat{H}=i g\left[\hat{\sigma}_{a}^{\dagger k} \hat{a}-\hat{a}^{\dagger} \hat{\sigma}_{a}^{k}+\hat{\sigma}_{b}^{\dagger k} \hat{b}-\hat{b}^{\dagger} \hat{\sigma}_{b}^{k}\right]+$

$+\frac{i \Omega}{2}\left[\hat{\sigma}_{c}^{\dagger k}-\hat{\sigma}_{c}^{k}\right]$,

where $g$ is the coupling constant between the atom and cavity mode $a$ or $b$, and $\hat{a}$ and $\hat{b}$ are the annihilation operators for light modes $a$ and $b$. Here, $\Omega=2 \varepsilon \lambda$, in which $\varepsilon$ considered to be real and constant, is the amplitude of the driving coherent light, and $\lambda$ is the coupling constant between the driving coherent light and the three-level atom. We also define that $\hat{\sigma}_{a}^{k}=|b\rangle_{k k}\left\langle a\left|, \hat{\sigma}_{b}^{k}=\right| c\right\rangle_{k k}\langle b|$, and $\hat{\sigma}_{c}^{k}=|c\rangle_{k k}\langle a|$ are lowering atomic operators. Following the procedure described in [24], the master equation for a coherently driven nondegenerate three-level atom, being in an open cavity and coupled to a two-mode vacuum reservoir, is found to be

$\frac{d}{d t} \hat{\rho}(t)=g\left[\hat{\sigma}_{a}^{\dagger k} \hat{a} \hat{\rho}-\hat{a}^{\dagger} \hat{\sigma}_{a}^{k} \hat{\rho}+\hat{\sigma}_{b}^{\dagger k} \hat{b} \hat{\rho}-\hat{b}^{\dagger} \hat{\sigma}_{b}^{k} \hat{\rho}-\hat{\rho} \hat{\sigma}_{a}^{\dagger k} \hat{a}+\right.$

$\left.+\hat{\rho} \hat{a}^{\dagger} \hat{\sigma}_{a}^{k}-\hat{\rho} \hat{\sigma}_{b}^{\dagger k} \hat{b}+\hat{\rho} \hat{b}^{\dagger} \hat{\sigma}_{b}^{k}\right]+\frac{\Omega}{2}\left[\hat{\sigma}_{c}^{\dagger k} \hat{\rho}-\hat{\sigma}_{c}^{k} \hat{\rho}+\right.$

$\left.+\hat{\rho} \hat{\sigma}_{c}^{\dagger k}-\hat{\rho} \hat{\sigma}_{c}^{k}\right]+\frac{\gamma}{2}\left[2 \hat{\sigma}_{b}^{k} \hat{\rho} \hat{\sigma}_{b}^{\dagger k}-\hat{\sigma}_{b}^{\dagger k} \hat{\sigma}_{b}^{k} \hat{\rho}-\hat{\rho} \hat{\sigma}_{b}^{\dagger k} \hat{\sigma}_{b}^{k}\right]+$ $+\frac{\gamma}{2}\left[2 \hat{\sigma}_{c}^{k} \hat{\rho} \hat{\sigma}_{c}^{\dagger k}-\hat{\sigma}_{c}^{\dagger k} \hat{\sigma}_{c}^{k} \hat{\rho}-\hat{\rho} \hat{\sigma}_{c}^{\dagger k} \hat{\sigma}_{c}^{k}\right]$

where $\gamma$ is the spontaneous emission decay constant.

Due to the normal ordering of the noise operators associated with the vacuum reservoir, the noise operators will not have any effect on the dynamics of the cavity mode operators [22-24]. Therefore, with the help of expression (1), one can drop the noise operators and write the quantum Langevin equations as

$\frac{d \hat{a}}{d t}=-\frac{\kappa}{2} \hat{a}-g \hat{\sigma}_{a}^{k}$,
$\frac{d \hat{b}}{d t}=-\frac{\kappa}{2} \hat{b}-g \hat{\sigma}_{b}^{k}$.

where $\kappa$ is the cavity damping constant.

\section{Equations of Evolution of Atomic Oprators}

Employing the relation $\frac{d}{d t}\langle\hat{A}\rangle=\operatorname{Tr}\left(\frac{d \hat{\rho}}{d t} \hat{A}\right)$ along with result (2), one can readily establish that

$\frac{d}{d t}\left\langle\hat{\sigma}_{a}^{k}\right\rangle=g\left(\left\langle\hat{\eta}_{b}^{k} \hat{a}\right\rangle-\left\langle\hat{\eta}_{a}^{k} \hat{a}\right\rangle+\left\langle\hat{b}^{\dagger} \hat{\sigma}_{c}^{k}\right\rangle\right)+\frac{\Omega}{2}\left\langle\hat{\sigma}_{b}^{\dagger k}\right\rangle-\gamma\left\langle\hat{\sigma}_{a}^{k}\right\rangle$,

$\frac{d}{d t}\left\langle\hat{\sigma}_{b}^{k}\right\rangle=g\left(\left\langle\hat{\eta}_{c}^{k} \hat{b}\right\rangle-\left\langle\hat{a}^{\dagger} \hat{\sigma}_{c}^{k}\right\rangle-\left\langle\hat{\eta}_{b}^{k} \hat{b}\right\rangle\right)-\frac{\Omega}{2}\left\langle\hat{\sigma}_{a}^{\dagger k}\right\rangle-\frac{\gamma}{2}\left\langle\hat{\sigma}_{a}^{k}\right\rangle$,

$\frac{d}{d t}\left\langle\hat{\sigma}_{c}^{k}\right\rangle=g\left(\left\langle\hat{\sigma}_{b}^{k} \hat{a}\right\rangle-\left\langle\hat{\sigma}_{a}^{k} \hat{b}\right\rangle\right)-\frac{\gamma}{2}\left\langle\hat{\sigma}_{c}^{k}\right\rangle+\frac{\Omega}{2}\left[\left\langle\hat{\eta}_{c}^{k}\right\rangle-\left\langle\hat{\eta}_{a}^{k}\right\rangle\right]$,

$\frac{d}{d t}\left\langle\hat{\eta}_{a}^{k}\right\rangle=g\left(\left\langle\hat{\sigma}_{a}^{\dagger k} \hat{a}\right\rangle+\left\langle\hat{a}^{\dagger} \hat{\sigma}_{a}^{k}\right\rangle\right)-\gamma\left\langle\hat{\eta}_{a}^{k}\right\rangle+\frac{\Omega}{2}\left[\left\langle\hat{\sigma}_{c}^{k}\right\rangle+\left\langle\hat{\sigma}_{c}^{\dagger k}\right\rangle\right]$,

$\frac{d}{d t}\left\langle\hat{\eta}_{b}^{k}\right\rangle=g\left(\left\langle\hat{\sigma}_{b}^{\dagger} \hat{b}\right\rangle-\left\langle\hat{\sigma}_{a}^{\dagger k} \hat{a}\right\rangle-\left\langle\hat{a}^{\dagger} \hat{\sigma}_{a}^{k}\right\rangle+\left\langle\hat{b}^{\dagger} \hat{\sigma}_{b}^{k}\right\rangle\right)-\gamma\left\langle\hat{\eta}_{b}^{k}\right\rangle$,

where $\hat{\eta}_{a}^{k}=|a\rangle_{k k}\left\langle a\left|, \hat{\eta}_{b}^{k}=\right| b\right\rangle_{k k}\langle b|$, and $\hat{\eta}_{c}^{k}=|c\rangle_{k k}\langle c|$.

We note that expressions (5)-(9) are nonlinear coupled differential equations. Therefore, it is not possible to obtain exact solutions. Then, employing the large-time approximation scheme for Eqs. (3) and (4), we obtain

$\hat{a}=-\frac{2 g}{\kappa} \hat{\sigma}_{a}^{k}$

ISSN 2071-0194. Ukr. J. Phys. 2018. Vol. 63, No. 11 
$\hat{b}=-\frac{2 g}{\kappa} \hat{\sigma}_{b}^{k}$.

Now, introducing Eqs. (10) and (11) into (5)-(9) and summing over $N$ three-level atoms, we get

$\frac{d}{d t}\left\langle\hat{m}_{a}\right\rangle=-\left(\gamma+\gamma_{c}\right)\left\langle\hat{m}_{a}\right\rangle+\frac{\Omega}{2}\left\langle\hat{m}_{b}^{\dagger}\right\rangle$,

$\frac{d}{d t}\left\langle\hat{m}_{b}\right\rangle=-\frac{1}{2}\left(\gamma+\gamma_{c}\right)\left\langle\hat{m}_{b}\right\rangle-\frac{\Omega}{2}\left\langle\hat{m}_{a}^{\dagger}\right\rangle$,

$\frac{d}{d t}\left\langle\hat{m}_{c}\right\rangle=-\frac{1}{2}\left(\gamma+\gamma_{c}\right)\left\langle\hat{m}_{c}\right\rangle+\frac{\Omega}{2}\left[\left\langle\hat{N}_{c}\right\rangle-\left\langle\hat{N}_{a}\right\rangle\right]$

$\frac{d}{d t}\left\langle\hat{N}_{a}\right\rangle=-\left(\gamma+\gamma_{c}\right)\left\langle\hat{N}_{a}\right\rangle+\frac{\Omega}{2}\left[\left\langle\hat{m}_{c}\right\rangle+\left\langle\hat{m}_{c}^{\dagger}\right\rangle\right]$

$\frac{d}{d t}\left\langle\hat{N}_{b}\right\rangle=-\left(\gamma+\gamma_{c}\right)\left\langle\hat{N}_{b}\right\rangle+\gamma_{c}\left\langle\hat{N}_{a}\right\rangle$,

in which $\gamma_{c}=\frac{4 g^{2}}{\kappa}$ is the stimulated emission decay constant, $\hat{m}_{a}=\sum_{k=1}^{N} \hat{\sigma}_{a}^{k}, \hat{m}_{b}=\sum_{k=1}^{N} \hat{\sigma}_{b}^{k}$, $\hat{m}_{c}=\sum_{k=1}^{N} \hat{\sigma}_{c}^{k}, \hat{N}_{a}=\sum_{k=1}^{N} \hat{\eta}_{a}^{k}, \hat{N}_{b}=\sum_{k=1}^{N} \hat{\eta}_{b}^{k}$, $\hat{N}_{c}=\sum_{k=1}^{N} \hat{\eta}_{c}^{k}$, with the operators $\hat{N}_{a}, \hat{N}_{b}$, and $\hat{N}_{c}$ representing the number of atoms in the top, intermediate, and bottom levels, respectively. In addition, employing the completeness relation $\hat{I}=\hat{\eta}_{a}^{k}+\hat{\eta}_{b}^{k}+\hat{\eta}_{c}^{k}$, we get

$\left\langle\hat{N}_{a}\right\rangle+\left\langle\hat{N}_{b}\right\rangle+\left\langle\hat{N}_{c}\right\rangle=N$.

Furthermore, using the definition and setting

$\hat{\sigma}_{a}^{k}=|b\rangle\langle a|$

for any $k$, we have

$\hat{m}_{a}=N|b\rangle\langle a|$.

One can find, following the same procedure described above, that $\hat{m}_{b}=N|c\rangle\left\langle b\left|, \hat{m}_{c}=N\right| c\right\rangle\langle a|, \hat{N}_{a}=$ $=N|a\rangle\left\langle a\left|, \hat{N}_{b}=N\right| b\right\rangle\left\langle b\left|, \hat{N}_{c}=N\right| c\right\rangle\langle c|$. Moreover, using the definition $\hat{m}=\hat{m}_{a}+\hat{m}_{b}$ and taking this result into account, we observe that $\hat{m}^{\dagger} \hat{m}=N\left(\hat{N}_{a}+\right.$ $\left.+\hat{N}_{b}\right), \hat{m} \hat{m}^{\dagger}=N\left(\hat{N}_{b}+\hat{N}_{c}\right)$, and $\hat{m}^{2}=N \hat{m}_{c}$. For $N$ three-level atoms, Eqs. (3) and (4) can be rewritten as

$$
\begin{aligned}
& \frac{d \hat{a}}{d t}=-\frac{\kappa}{2} \hat{a}+\lambda^{\prime} \hat{m}_{a}, \\
& \frac{d \hat{b}}{d t}=-\frac{\kappa}{2} \hat{b}+\lambda^{\prime \prime} \hat{m}_{b},
\end{aligned}
$$

where $\lambda^{\prime}$ and $\lambda^{\prime \prime}$ are constants whose values remain to be determined. Furthermore, using Eqs. (10) and ISSN 2071-0194. Ukr. J. Phys. 2018. Vol. 63, No. 11
(11) and summing over all atoms, we obtain the commutation relations of the cavity mode operators:

$\left[\hat{a}, \hat{a}^{\dagger}\right]=\frac{\gamma_{c}}{\kappa}\left[\hat{N}_{b}-\hat{N}_{a}\right]$,

$\left[\hat{b}, \hat{b}^{\dagger}\right]=\frac{\gamma_{c}}{\kappa}\left[\hat{N}_{c}-\hat{N}_{b}\right]$.

Here, $\left[\hat{a}, \hat{a}^{\dagger}\right]=\sum_{k=1}^{N}\left[\hat{a}, \hat{a}^{\dagger}\right]_{k}$, and $\left[\hat{b}, \hat{b}^{\dagger}\right]=\sum_{k=1}^{N}\left[\hat{b}, \hat{b}^{\dagger}\right]_{k}$ stand for the commutators $\hat{a}$ and $\hat{a}^{\dagger}$, and $\hat{b}$ and $\hat{b}^{\dagger}$. On the other hand, employing the steady-state solutions of (20) and (21), one can easily verify that

$\left[\hat{a}, \hat{a}^{\dagger}\right]=N\left(\frac{2 \lambda^{\prime}}{\kappa}\right)^{2}\left(\hat{N}_{b}-\hat{N}_{a}\right)$,
$\left[\hat{b}, \hat{b}^{\dagger}\right]=N\left(\frac{2 \lambda^{\prime \prime}}{\kappa}\right)^{2}\left(\hat{N}_{c}-\hat{N}_{b}\right)$.

Thus, the inspection of Eqs. (22) and(23) with (24) and (25) shows that

$\lambda^{\prime}=\lambda^{\prime \prime}= \pm \frac{g}{\sqrt{N}}$.

Hence in view of this result, Eqs. (24) and (25) can be rewritten as

$\frac{d \hat{a}}{d t}=-\frac{\kappa}{2} \hat{a}+\frac{g}{\sqrt{N}} \hat{m}_{a}$,
$\frac{d \hat{b}}{d t}=-\frac{\kappa}{2} \hat{b}+\frac{g}{\sqrt{N}} \hat{m}_{b}$.

Now, adding Eqs. (22) and (23) and summing (27) and (28), we get

$\left[\hat{c}, \hat{c}^{\dagger}\right]=\frac{\gamma_{c}}{\kappa}\left[\hat{N}_{c}-\hat{N}_{a}\right]$,

$\frac{d \hat{c}}{d t}=-\frac{\kappa}{2} \hat{c}+\frac{g}{\sqrt{N}} \hat{m}$,

where $\hat{c}=\hat{a}+\hat{b}$.

Furthermore, applying the large-time approximation scheme to Eqs. (12) and (13), one can obtain $\left\langle\hat{m}_{a}(t)\right\rangle=\left\langle\hat{m}_{b}(t)\right\rangle=0$. Hence, the steady-state solutions of the expectation values of the cavity mode operators described in Eqs. (27), (28), and (30) are found to be $\langle\hat{a}(t)\rangle_{s s}=\langle\hat{b}(t)\rangle_{s s}=\langle\hat{a}(t)\rangle_{s s}=0$. Therefore, in view of the linear equations described by expressions (27), (28), and (30) with the corresponding solutions, we claim that $\hat{a}(t), \hat{b}(t)$, and $\hat{c}(t)$ are Gaussian variables with zero means. 


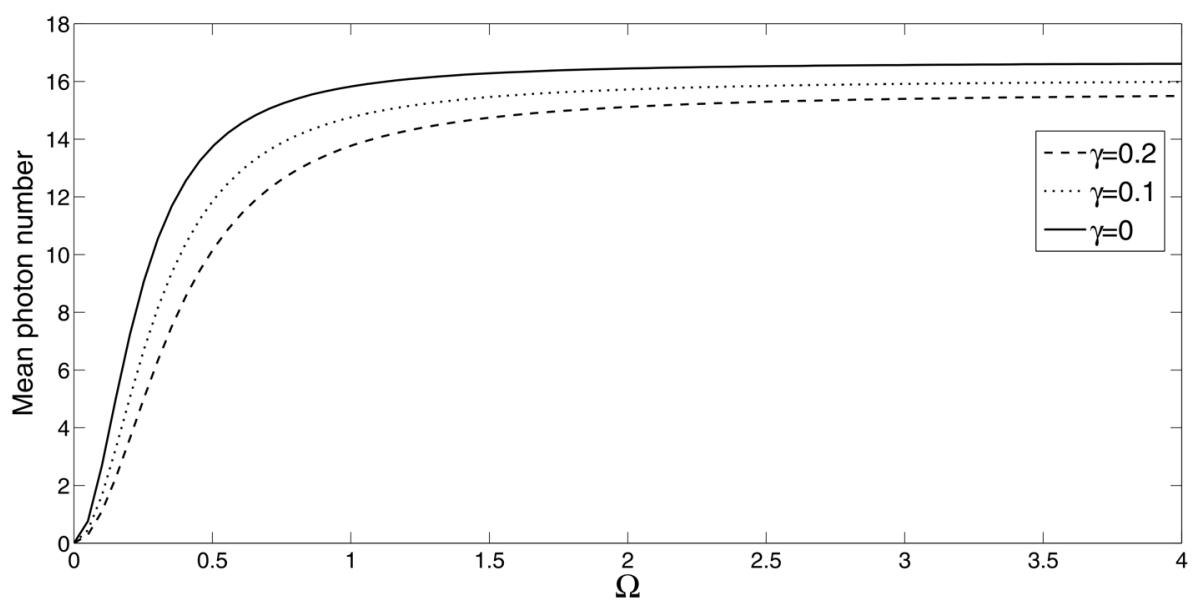

Fig. 1. Plots of the mean photon number versus $\Omega$ for $\gamma_{c}=0.4, \kappa=0.8, N=50$, and for different values of $\gamma$

In addition, the steady-state solutions of the atomic operators are found to be

$$
\begin{aligned}
\left\langle\hat{N}_{a}\right\rangle_{s s} & =\left[\frac{\left(\gamma_{c}+\gamma\right) \Omega^{2}}{\left(\gamma_{c}+\gamma\right)^{3}+\left(3 \gamma_{c}+2 \gamma\right) \Omega^{2}}\right] N, \\
\left\langle\hat{N}_{b}\right\rangle_{s s} & =\left[\frac{\Omega^{2} \gamma_{c}}{\left(\gamma_{c}+\gamma\right)^{3}+\left(3 \gamma_{c}+2 \gamma\right) \Omega^{2}}\right] N, \\
\left\langle\hat{N}_{c}\right\rangle_{s s} & =\left[\frac{\left(\gamma_{c}+\gamma\right)^{3}+\Omega^{2}\left(\gamma_{c}+\gamma\right)}{\left(\gamma_{c}+\gamma\right)^{3}+\left(3 \gamma_{c}+2 \gamma\right) \Omega^{2}}\right] N, \\
\left\langle\hat{m}_{c}\right\rangle_{s s} & =\left[\frac{\Omega\left(\gamma_{c}+\gamma\right)^{2}}{\left(\gamma_{c}+\gamma\right)^{3}+\left(3 \gamma_{c}+2 \gamma\right) \Omega^{2}}\right] N .
\end{aligned}
$$

Initially (when $\Omega=0$ ), all the atoms are on the lower level $\left(\left\langle\hat{N}_{c}\right\rangle_{s s}=N\right)$, while the number of atoms on the top and intermediate levels are zero.

\section{Mean Photon Number}

To learn about the brightness of the generated light, it is necessary to study the mean number of photon pairs describing the two-mode cavity radiation that can be defined as

$\bar{n}=\left\langle\hat{c}^{\dagger} \hat{c}\right\rangle$

where $\hat{c}$ is the annihilation operator of the twomode cavity. Employing the steady-state solution of Eq. (30), it can be found to be

$\bar{n}=\frac{\gamma_{c}}{k}\left[\left\langle\hat{N}_{a}\right\rangle_{s s}+\left\langle\hat{N}_{b}\right\rangle_{s s}\right]$.

972
With the aid of Eqs. (31) and (32), one can readily show that

$\bar{n}=\left(\frac{\gamma_{c}}{k} N\right)\left[\frac{\Omega^{2}\left(2 \gamma_{c}+\gamma\right)}{\left(\gamma_{c}+\gamma\right)^{3}+\left(3 \gamma_{c}+2 \gamma\right) \Omega^{2}}\right]$.

In the case where the spontaneous emission is absent,

$\bar{n}=\frac{\gamma_{c}}{\kappa} N\left[\frac{2 \Omega^{2}}{\gamma_{c}^{2}+3 \Omega^{2}}\right]$.

It is not difficult to see for $\Omega \gg \gamma_{c}$ that

$\bar{n}=\frac{2 \gamma_{c}}{3 \kappa} N$

From Fig. 1, one can clearly see the mean photon number for the two-mode cavity light in the presence (when $\gamma \neq 0$ ) and in the absence of the spontaneous emission (when $\gamma=0$ ). Moreover, it is found that the mean photon number of the two-mode light increases with $\Omega$ in both cases. This indicates, on the other hand, that the mean photon number of the twomode light beam is greater when $\gamma=0$, than when $\gamma \neq 0$. Therefore, the presence of the spontaneous emission decreases the mean photon number.

\section{Quadrature Fluctuations}

In this section, the quadrature variance and quadrature squeezing of the two-mode light in the cavity produced by a two-mode three-level cascade laser is analyzed.

ISSN 2071-0194. Ukr. J. Phys. 2018. Vol. 63, No. 11 


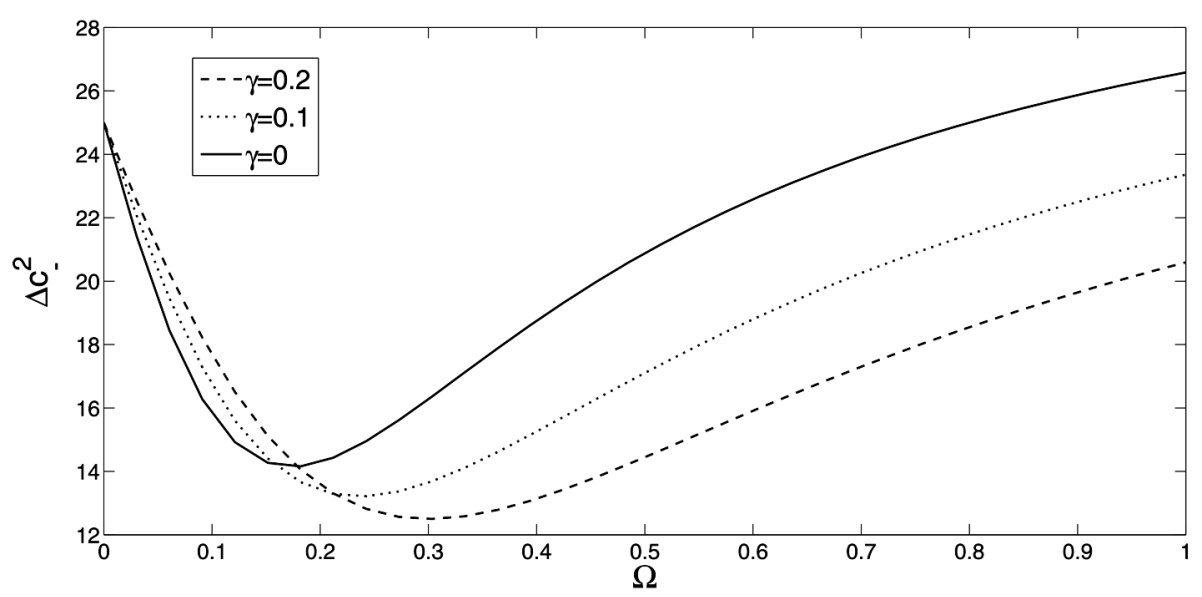

Fig. 2. Plots of $\left(\Delta c_{-}\right)^{2}$ versus $\Omega$ for $\gamma_{c}=0.4, k=0.8, N=50$ and for different values of $\gamma$

\subsection{Quadrature variance}

The squeezing properties of the two-mode cavity light are described by two quadrature operators

$\hat{c}_{+}=\hat{c}^{\dagger}+\hat{c}$

$\hat{c}_{-}=i\left(\hat{c}^{\dagger}-\hat{c}\right)$,

where $\hat{c}=\hat{a}+\hat{b}$, ,Making use of the well-known definition of the variance of an operator, the variances of the quadrature operators (40) and (41) are found to have the form

$\left(\Delta \hat{c}_{ \pm}\right)^{2}=\left\langle\hat{c} \hat{c}^{\dagger}\right\rangle+\left\langle\hat{c}^{\dagger} \hat{c}\right\rangle \pm\left\langle\hat{c}^{2}\right\rangle \pm\left\langle\hat{c}^{\dagger}\right\rangle \mp\langle\hat{c}\rangle^{2} \mp$

$\mp\left\langle\hat{c}^{\dagger}\right\rangle^{2}-2\langle\hat{c}\rangle\left\langle\hat{c}^{\dagger}\right\rangle$.

With the aid of the steady-state solution of Eq. (30), one can easily establish that

$\left(\Delta \hat{c}_{ \pm}\right)^{2}=\frac{\gamma_{c}}{k}\left[N+\left\langle\hat{N}_{b}\right\rangle_{s s} \pm 2\left\langle\hat{m}_{c}\right\rangle_{s s}\right]$.

This yields

$$
\begin{aligned}
& \left(\Delta \hat{c}_{ \pm}\right)^{2}=\frac{\gamma_{c}}{k} N\left[\frac{\left(\gamma_{c}+\gamma\right)^{3}+\left(4 \gamma_{c}+2 \gamma\right) \Omega^{2}}{\left(\gamma_{c}+\gamma\right)^{3}+\left(3 \gamma_{c}+2 \gamma\right) \Omega^{2}}\right] \pm \\
& \left. \pm \frac{2 \Omega\left(\gamma_{c}+\gamma\right)^{2}}{\left(\gamma_{c}+\gamma\right)^{3}+\left(3 \gamma_{c}+2 \gamma\right) \Omega^{2}}\right] .
\end{aligned}
$$

For $\gamma=0$, the quadrature variances take the form

$\left(\Delta \hat{c}_{ \pm}\right)^{2}=\frac{\gamma_{c}}{k} N\left[\frac{4 \Omega^{2}+\gamma_{c}^{2} \pm 2 \Omega \gamma_{c}}{\gamma_{c}^{2}+3 \Omega^{2}}\right]$.

ISSN 2071-0194. Ukr. J. Phys. 2018. Vol. 63, No. 11
For $\Omega \gg \gamma_{c}$,

$\left(\Delta \hat{c}_{ \pm}\right)^{2}=\frac{4 \gamma_{c}}{3 k} N=2 \bar{n}$

where $\bar{n}$ is given by Eq. (39). It can be seen that expression (46) represents the normally ordered quadrature variance for chaotic light. Moreover, in the case where the driving coherent light is absent, one can see that

$\left(\Delta \hat{c}_{+}\right)_{v}^{2}=\left(\Delta \hat{c}_{-}\right)_{v}^{2}=\frac{\gamma_{c}}{k} N$

which is the normally ordered quadrature variance of the two-mode cavity light in the vacuum state. It is also observed that the uncertainties in the plus and minus quadratures are equal and satisfy the minimum uncertainty relation.

\subsection{Quadrature squeezing}

The quadrature squeezing of the two-mode cavity light relative to the quadrature variance of the twomode vacuum light can be defined as

$S=\frac{\left(\Delta \hat{c}_{ \pm}\right)_{v}^{2}-\left(\Delta \hat{c}_{-}\right)^{2}}{\left(\Delta \hat{c}_{ \pm}\right)_{v}^{2}}$

where $\left(\Delta \hat{c}_{ \pm}\right)_{v}^{2}$ is the quadrature variance in the vacuum state given by Eq. (47). Taking Eqs. (44), (47), and (48) into account yields

$S=\frac{2 \Omega\left(\gamma_{c}+\gamma\right)^{2}-\Omega^{2} \gamma_{c}}{\left(\gamma_{c}+\gamma\right)^{3}+\left(3 \gamma_{c}+2 \gamma\right) \Omega^{2}}$ 


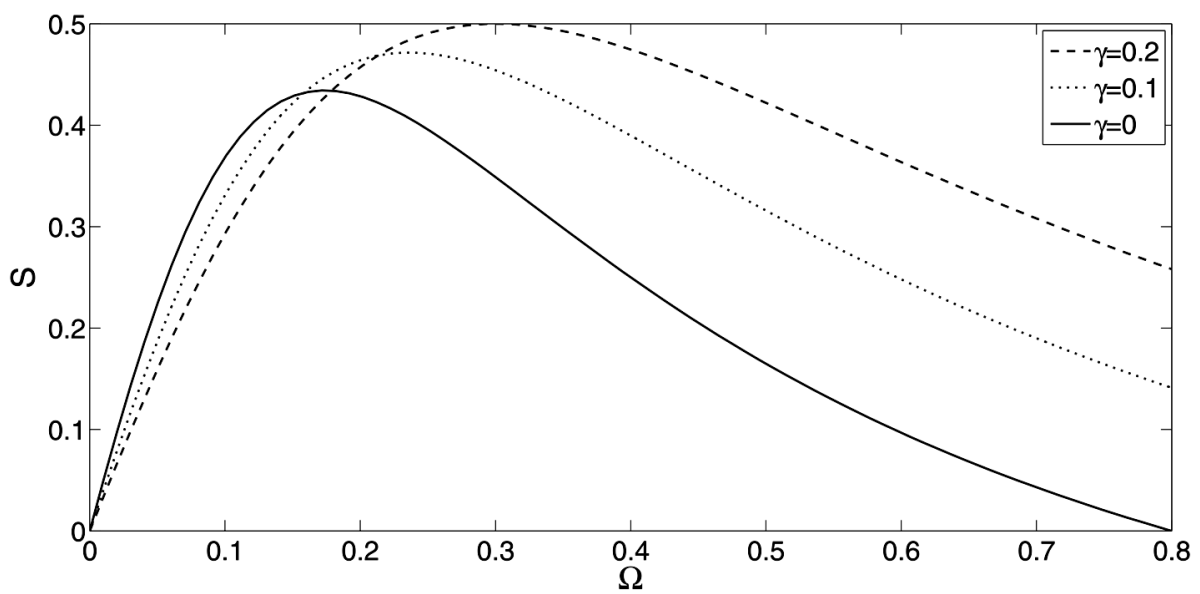

Fig. 3. Plots of the quadrature squeezing versus $\Omega$ for $\gamma_{c}=0.4$ and for different values of $\gamma$

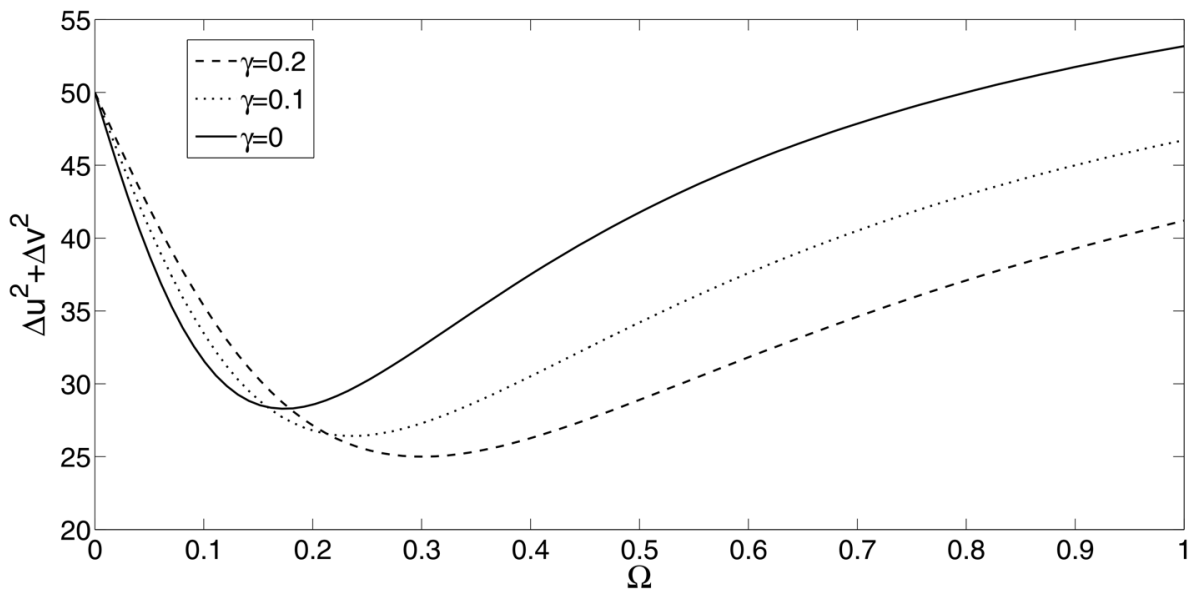

Fig. 4. Plot of the $\Delta \hat{u}^{2}+\Delta \hat{v}^{2}$ of the two-mode cavity light at the steady-state versus $\Omega$ for $\gamma_{c}=0.4, \kappa=0.8, N=50$, and for different values of $\gamma$

We observe that, in Eq. (49), the quadrature squeezing of the cavity light is independent of the number of photons.

It is indicated in Fig. 4 that the squeezing exhibits in a different manner from the two-mode cavity radiation, for values of $\Omega$ between 0 and 1 , with different degrees of squeezing. Moreover, it is possible to realize that the degree of squeezing increases with the spontaneous emission decay constant, $\gamma$. We have found the $50 \%$ degree of squeezing for $\gamma=0.2$ and $\gamma_{c}=0.4$. This occurs, when the three-level laser is operating at $\Omega=0.3$. Hence, one can observe that a light produced by a nondegenerate three-level laser can exhibit the equal degree of squeezing as compared, for example, with the light generated by a three-level laser in which the three-level atoms available in a closed cavity are pumped to the top level by means of the electron bombardment $[10,24]$.

\section{Entanglement Properties of the Two-Mode Light}

The quantum entanglement between the two cavity modes $a$ and $b$ proposed by Duan-Giedke-CiracZoller (DGCZ) in [25], where the sufficient condition for entangled quantum states is given. According to DGCZ, a quantum state of the system is said to be entangled, if the sum of the variances of the EPR-like quadrature operators, $\hat{u}$ and $\hat{v}$, satisfies the inequality

$(\Delta \hat{u})^{2}+(\Delta \hat{v})^{2}<2 N$,

ISSN 2071-0194. Ukr. J. Phys. 2018. Vol. 63, No. 11 
where

$\hat{u}=\hat{x}_{a}-\hat{x}_{b}, \quad \hat{v}=\hat{p}_{a}+\hat{p}_{b}$,

with $\hat{x}_{a}=\left(\hat{a}^{\dagger}+\hat{a}\right) / \sqrt{2}, \hat{x}_{b}=\left(\hat{b}^{\dagger}+\hat{b}\right) / \sqrt{2}, \hat{p}_{a}=$ $i\left(\hat{a}^{\dagger}-\hat{a}\right) / \sqrt{2}, \hat{p}_{b}=i\left(\hat{b}^{\dagger}-\hat{b}\right) / \sqrt{2}$ being the quadrature operators for modes $\hat{a}$ and $\hat{b}$.

Taking (51) into account, Eq. (50) yields

$\Delta \hat{u}^{2}+\Delta \hat{v}^{2}=2 \frac{\gamma_{c}}{\kappa}\left[N+\left\langle\hat{N}_{b}\right\rangle_{s s}-\left\langle\hat{m}_{c}\right\rangle_{s s}\right]$.

Thus, in view of equation (52) together with (43), the sum of the variances of $\hat{u}$ and $\hat{v}$ can be expressed as

$\Delta \hat{u}^{2}+\Delta \hat{v}^{2}=2 \Delta c_{-}^{2}$,

where $\Delta c_{-}^{2}$ is given by (43). One can readily see from this result that the degree of entanglement is directly proportional to the degree of squeezing of the twomode light.

One can immediately notice that this particular entanglement measure is directly related the twomode squeezing. This direct relationship shows that, whenever there is a two-mode squeezing in the system, there will be entanglement in the system as well. It is worth to note that the entanglement disappears when the squeezing vanishes. This is due to the fact that the entanglement is directly related to the squeezing, as given by (43). It also follows that, like the mean photon number and quadrature variance, the degree of entanglement depends on the number of atoms. With the help of criterion (50), we get that a significant entanglement occurs between the states of the light generated in the cavity. This is due to the strong correlation between the radiation emitted, when the atoms decay from the upper energy level to the lower via the intermediate level.

In the following, the sum of the variances of a pair of EPR-type operators $\Delta \hat{u}^{2}+\Delta \hat{v}^{2}$ is plotted against the amplitude of the driving coherent light so that the available entanglement is clearly evident for various values of the spontaneous emission rate, $\gamma$.

\section{Normalized Second-Order Correlation Functions}

The second-order correlation function of the separate mode, as well as for the superposition of the two modes of the cavity radiation at equal time, can also be investigated, by using $[26,27]$ :

$g_{(a, b)}^{(2)}(0)=\frac{\left\langle\hat{a}^{\dagger} \hat{a} \hat{b}^{\dagger} \hat{b}\right\rangle}{\left\langle\hat{a}^{\dagger} \hat{a}\right\rangle\left\langle\hat{b}^{\dagger} \hat{b}\right\rangle}$.

Since $\hat{a}$ and $\hat{b}$ are Gaussian variables with vanishing means, the normalized second-order correlation function for the two-mode light takes, at the steady-state, the form

$g_{(a, b)}^{(2)}(0)=1+\frac{\langle\hat{b} \hat{a}\rangle\left\langle\hat{a}^{\dagger} \hat{b}^{\dagger}\right\rangle}{\left\langle\hat{a}^{\dagger} \hat{a}\right\rangle\left\langle\hat{b}^{\dagger} \hat{b}\right\rangle}$.

It then follows that

$g_{(a, b)}^{(2)}(0)=1+\frac{\left\langle\hat{m}_{c}\right\rangle_{s s}^{2}}{\left\langle\hat{N}_{a}\right\rangle_{s s}\left\langle\hat{N}_{b}\right\rangle_{s s}}$.

In view of (31), (32), and (34), we obtain

$g_{(a, b)}^{(2)}(0)=1+\frac{\left(\gamma_{c}+\gamma\right)^{3}}{\gamma_{c} \Omega^{2}}$

It can be seen from this result that the second-order correlation function of the two-mode light does not depend on the number of atoms.

Figure 5 shows that the second-order correlation function for the two-mode light versus $\Omega$ in the presence $(\gamma \neq 0)$ and absence $(\gamma=0)$ of the spontaneous emission. One can see from this figure that $g_{(a, b)}^{(2)}(0)$ decreases, as $\Omega$ increases in both cases. It can be observed from the same figure that the second-order correlation function vanishes for $\Omega<0.05$. Moreover, the effect of the spontaneous emission increases the second-order correlation function.

Now, it is essential to calculate the second-order correlation function for the individual mode to have an insight for the previous result. To this end, the second-order correlation function for mode $a$ is given by

$g_{(a, a)}^{(2)}(0)=\frac{\left\langle: \hat{n}_{a} \hat{n}_{a}:\right\rangle}{\left\langle\hat{n}_{a}\right\rangle^{2}}$

where :: represent normal ordering and $\hat{n}_{a}=\hat{a}^{\dagger} \hat{a}$ is the photon number operator for mode $a$. Since $\hat{a}$ is a Gaussian variable with vanishing mean, one can easily verify that

$g_{(a, a)}^{(2)}(0)=2$. 


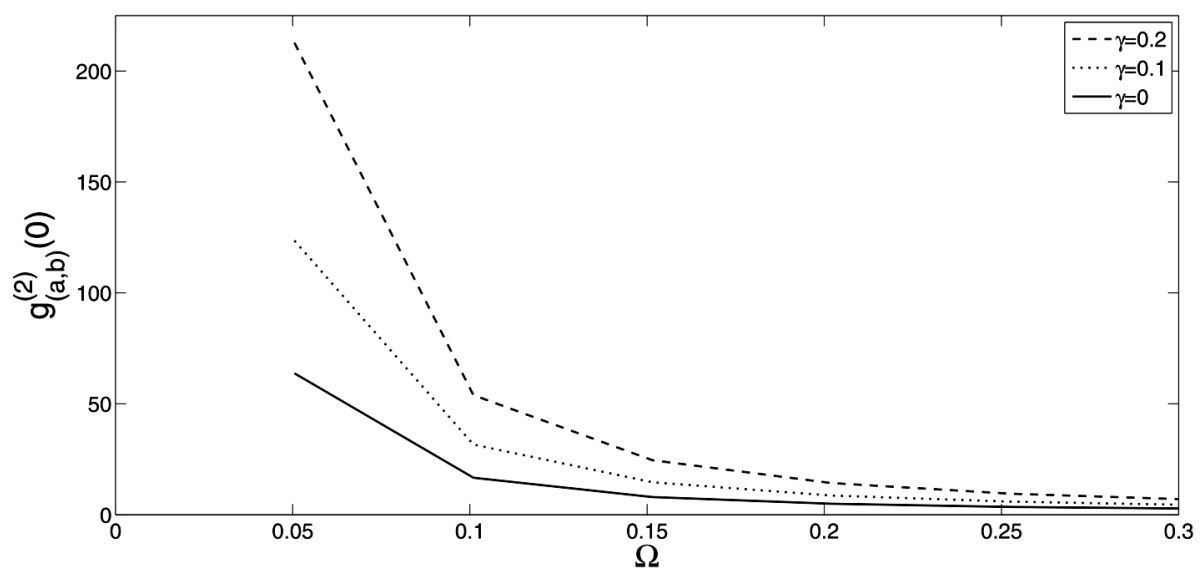

Fig. 5. Plot of the $g_{(a, b)}^{(2)}(0)$ of the two-mode cavity light versus $\Omega$ for $\gamma_{c}=0.4$ and for different values of $\gamma$

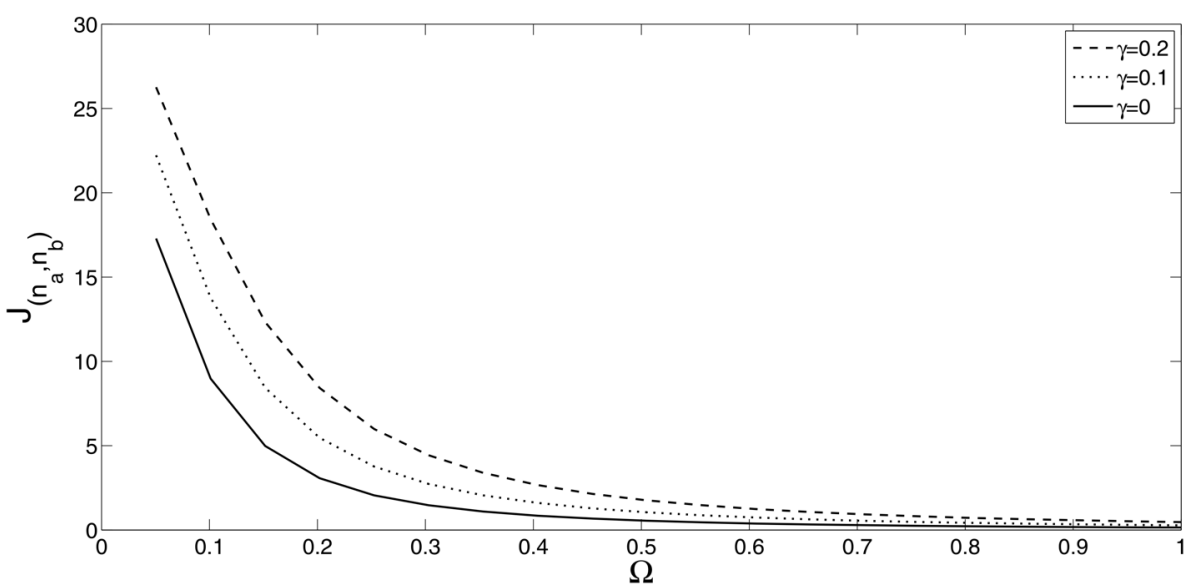

Fig. 6. Plot of the $J_{\left(\hat{n}_{a}, \hat{n}_{b}\right)}$ of the two-mode cavity light at the steady-state versus $\Omega$ for $\gamma_{c}=0.4, \kappa=0.8, N=50$, and for different values of $\gamma$

Similarly, the second-order correlation function for mode $b$ is found to be

$g_{(b, b)}^{(2)}(0)=2$.

From expressions (59) and (60), we get the secondorder correlation function for light in a chaotic state. So, the cavity modes $a$ and $b$ are separately in a chaotic or thermal state.

Furthermore, in order to quantify the correlation between the two modes, we introduce the linear correlation coefficient in terms of a covariance as [28]

$J_{\left(\hat{n}_{a}, \hat{n}_{b}\right)}=\frac{\operatorname{cov}\left(\hat{n}_{a}, \hat{n}_{b}\right)}{\sqrt{\Delta \hat{n}_{a}^{2}} \sqrt{\Delta \hat{n}_{b}^{2}}}$, where $\Delta \hat{n}_{a}^{2}$ and $\Delta \hat{n}_{b}^{2}$ are the variances of the photon number for modes $a$ and $b$, respectively. So, the covariance of the photon numbers is defined by

$\operatorname{cov}\left(\hat{n}_{a}, \hat{n}_{b}\right)=\left\langle\hat{n}_{a} \hat{n}_{b}\right\rangle-\left\langle\hat{n}_{a}\right\rangle\left\langle\hat{n}_{b}\right\rangle$

One can easily verify, using the fact that $\hat{a}$ and $\hat{b}$ are Gaussian variables, in the steady state that

$\operatorname{cov}\left(\hat{n}_{a}, \hat{n}_{b}\right)=\langle\hat{b} \hat{a}\rangle_{s s}\left\langle\hat{a}^{\dagger} \hat{b}^{\dagger}\right\rangle_{s s}$.

Since the cavity modes are separately in a chaotic state, the variances of the photon numbers obey the relation for a chaotic state, $\Delta \hat{n}_{a}^{2}=\left\langle\hat{n}_{a}\right\rangle+\left\langle\hat{n}_{a}\right\rangle^{2}$ and $\Delta \hat{n}_{b}^{2}=\left\langle\hat{n}_{b}\right\rangle+\left\langle\hat{n}_{b}\right\rangle^{2}$. Accounting for this fact and (63),

ISSN 2071-0194. Ukr. J. Phys. 2018. Vol. 63, No. 11 
the correlation function can be rewritten as

$$
J_{\left(\hat{n}_{a}, \hat{n}_{b}\right)}=\frac{\langle\hat{b} \hat{a}\rangle_{s s}\left\langle\hat{a}^{\dagger} \hat{b}^{\dagger}\right\rangle_{s s}}{\sqrt{\left\langle\hat{n}_{a}\right\rangle_{s s}+\left\langle\hat{n}_{a}\right\rangle_{s s}^{2}} \sqrt{\left\langle\hat{n}_{b}\right\rangle_{s s}+\left\langle\hat{n}_{b}\right\rangle_{s s}^{2}}} .
$$

In Fig. 6, the linear correlation coefficient versus the amplitude of the driving coherent light, $\Omega$, is plotted. It is also found from this figure that, for $\Omega$ very close to 0 , the intermode correlation would be significantly large, since the mean photon number of the light in mode $b$ is very close to zero, when almost all atoms initially occupy in the lower level. Moreover, similar to the second-order correlation function, the plots in Fig. 6 show that the linear correlation coefficient vanishes, when $\Omega<0.05$.

\section{Conclusion}

In conclusion, the squeezing and entanglement properties of a non-degenerate three-level laser driven by coherent light and coupled to a two-mode vacuum reservoir via a single-port mirror, whose open cavity contains $N$ non-degenerate three-level atoms, are thoroughly analyzed. We have carried out the analysis by putting the noise operators associated with the vacuum reservoir in the normal order and by considering the interaction of the three-level atoms with the vacuum reservoir outside the cavity. The master equation and the quantum Langevin equations for the cavity light are obtained. Applying these equations, the equations of evolution of the cavity mode and the atomic operators are solved. Making use of the steady-state solutions of atomic and cavity mode operators, the quadrature variance, quadrature squeezing, and entanglement for the two-mode cavity light, at the steady state, are determined. In addition, the normalized second-order correlation function is obtained for the individual mode, as well as for the superposition of the two modes. Finally, the linear correlation coefficient between the two modes is obtained.

The analysis showed that the intracavity quadrature squeezing is enhanced due to the spontaneous emission. It is found that the squeezing and entanglement in the two-mode light are directly related. As a result, an increase in the degree of squeezing directly leads to an increase in the degree of entanglement and vice versa. This shows that, whenever there is squeezing in the two-mode light, there exists an entanglement in the system. In addition, it is shown that the photons in the laser cavity are highly correlated and the degree of photon number correlation increases with the spontaneous emission decay constant, $\gamma$. Therefore, the presence of the spontaneous emission leads to an increase in the degree of entanglement, squeezing, and photon number correlation.

1. J.M. Liu, B.S. Shi, X.F. Fan, J. Li, G.C. Guo. Wigner function description of continuous variable entanglement swapping. J. Opt. B: Quant. Semiclass. Opt. 3, 189 (2001).

2. S.L. Braunstein, H.J. Kimble. Dense coding for continuous variables. Phys. Rev. A 61, 42302 (2000).

3. S. Lloyd and S.L. Braunstein. Quantum computation over continuous variables. Phys. Rev. Lett. 82, 1784 (1999).

4. S.L. Braunstein. Quantum error correction for communication with linear optics. Nature 394, 47 (1998).

5. T.C. Ralph. Continuous variable quantum cryptography. Phys. Rev. A 61, 010302 (2000).

6. A. Einstein, B. Podolsky, R. Rosen. Can quantummechanical description of physical reality be considered complete? Phys. Rev. 47, 777 (1935).

7. M.O. Scully, K. Wodkiewicz, M.S. Zubairy, J. Bergou, N. Lu, J. Meyer ter Vehn. Two-photon correlated-spontaneous-emission laser: Quantum noise quenching and squeezing. Phys. Rev. Lett. 60, 1832 (1988).

8. E. Alebachew. Enhanced squeezing and entanglement in a non-degenerate three-level cascade laser with injected squeezed light. Opt. Commun. 280, 133 (2007).

9. T. Abebe. Enhancement of squeezing and entanglement in a non-degenerate three-level cascade laser with coherently driven cavity. Ukr. J. Phys. 63, No. 8, 733 (2018).

10. N. Lu, F.X. Zhao, J. Bergou. Nonlinear theory of a twophoton correlated-spontaneous-emission laser: A coherently pumped two-level-two-photon laser. Phys. Rev. A 39, 5189 (1989).

11. N. Lu, S.Y. Zhu. Quantum theory of two-photon correlated-spontaneous-emission lasers: Exact atom-field interaction Hamiltonian approach. Phys. Rev. A 40, 5735 (1989).

12. C.A. Blockley, D.F. Walls. Intensity fluctuations in a frequency down-conversion process with three-level atoms Phys. Rev. A 43, 5049 (1991).

13. N.A. Ansari, J. Gea-Banacloche, M.S. Zubairy. Phasesensitive amplification in a three-level atomic system. Phys. Rev. A 41, 5179 (1990).

14. N.A. Ansari. Effect of atomic coherence on the second-and higher-order squeezing in a two-photon three-level cascade atomic system. Phys. Rev. A 48, 4686 (1993).

15. J. Anwar, M.S. Zubairy. Quantum-statistical properties of noise in a phase-sensitive linear amplifier. Phys. Rev. A 49, 481 (1994).

16. N.A. Ansari. Theory of a two-mode phase-sensitive amplifier. Phys. Rev. A 46, 1560 (1992). 
17. H. Xiong, M.O. Scully, M.S. Zubairy. Correlated spontaneous emission laser as an entanglement amplifier. Phys. Rev. Lett. 94, 023601 (2005).

18. C.M. Caves, B.L. Schumaker. New formalism for twophoton quantum optics. I. Quadrature phases and squeezed states. Phys. Rev. A 31, 3068 (1985).

19. C.J. Villas-Boas, M.H.Y. Moussa. One-step generation of high-quality squeezed and EPR states in cavity QED. Eur. Phys. J. D 32, 147 (2005).

20. S. Tesfa. Entanglement amplification in a nondegenerate three-level cascade laser. Phys. Rev. A 74, 043816 (2006).

21. E. Alebachew, K. Fesseha. Coherently driven three-level laser with parametric amplifier. Opt. Commun. 265, 314 (2006).

22. F. Kassahun. Three-level laser dynamics with the atoms pumped by electron bombardment. https:// arXiv:1105.1438v3 quant-ph (2012).

23. F. Kassahun. Stimulated emission by two-level atoms pumped to the upper level. Opt. Commun. 284, 1357 (2011).

24. F. Kassahun. Refined Quantum Analysis of Light (CreateSpace Independent Publ. Platform, 2014).

25. L.M. Duan, G. Giedke, J.I. Cirac, P. Zoller. Inseparability criterion for continuous variable systems. Phys. Rev. Lett. 84, $2722(2000)$

26. M.O. Scully, M.S. Zubairy. Quantum Optics (Cambridge Univ. Press, 1997).
27. C. Gerry, P.L. Knight. Introductory Quantum Optics (Cambridge Univ. Press, 2005).

28. D.F. Walls, J.G. Milburn. Quantum Optics (Springer, 1994).

Received 13.09.18

T. Абебе

КВАНТОВИЙ АНАЛІЗ НЕВИРОДЖЕНОГО

ТРИРІВНЕВОГО ЛАЗЕРА ЗІ СПОНТАННИМ

ВИПРОМІНЮВАННЯМ І ВАКУУМНИМ

РЕЗЕРВУАРОМ ЗА ВІДСУТНІСТЮ ШУМУ

$\mathrm{P}$ е $з$ ю м е

Аналізуються квантові властивості світла резонатора від когерентно збудженого невиродженого трирівневого лазера з відкритим резонатором. Лазер взаємодіє з двомодовим вакуумним резервуаром, для якого враховується нормальне впорядкування операторів шуму. 3 використанням квантових рівнянь Ланжевена для операторів мод резонатора i рішень еволюційних рівнянь для очікуваних величин атомних операторів описані властивості стиснення, посилення змішування і нормалізована другого порядку кореляційна функція випромінювання резонатора. За певних умов трирівневий лазер генерує стисле світло з максимальним стисненням у резонаторі рівним 50\% нижче рівня вакуумного стану. Виявлено, що присутність спонтанного випромінювання підсилює квадратурне стиснення і переплутування i зменшує середнє число фотонів, випромінюваних двомодовим резервуаром. 